\title{
Mayores y nuevas tecnologías: motivaciones y dificultades
}

\author{
Liberto Macías González \\ Universitat Oberta per a Majors (UOM). Universitat de les Illes Balears, España \\ liberto.macias@uib.es \\ Cristina Manresa-Yee \\ Departament de Ciències Matemàtiques i Informàtica. Universitat de les Illes Balears, España
}

\begin{abstract}
Students participating in University Programs for Seniors are gradually more adapted to the technological changes taking place in society. E-inclusion is becoming a reality and the sentence "This is not for me" is less heard. We are concerned in knowing the motivations that make students enroll in an educational program like the University Programs for Seniors and the reasons that make students participate in a massive way in workshops or courses related to technology issues. The needs and interests shown by the students help program managers to plan appropriate activities and teachers to know better the kind of student that attends their classes. It is important to know the difficulties that our students confront, as it will help us to adapt the class contents to the group and to transform their weaknesses into strengths. Therefore, our case study analyses the motivations and interests of the group of students that is currently attending a course related with new technologies in the Open University for Seniors (UOM). The work also gathers together the main common difficulties when working with the computer. Finally, results are compared with a group of students that have never enrolled in a course related with new technologies.
\end{abstract}

Keywords: elderly people, new technologies, University program and education

\section{Resumen}

Los alumnos participantes en programas universitarios para mayores (PUM) cada vez están más adaptados a los cambios tecnológicos que se están produciendo de manera progresiva en la sociedad. La e-inclusión es cada vez más real y el argumento de "eso no es para mí» cada vez lo escuchamos menos. Pero el tema que más nos interesa y al que le queremos dar el espacio que merece en este estudio es el conocer cuáles son las motivaciones que hacen que, primero, se matriculen en un programa educativo, como los programas universitarios para mayores, $y$, segundo, que se matriculen de una manera masiva a los talleres, o cursos, relacionados con temas tecnológicos en sus diversas variantes. Las necesidades y los intereses que los alumnos muestran ayudan a los responsables de los programas a crear actividades idóneas para los alumnos mayores y a los docentes a conocer un poco más al alumnado que acude a sus clases. Conocer las dificultades con las que nuestros alumnos se encuentran es importante debido a que, de esta manera, podemos adaptar los contenidos al grupo y así posibilitar que esas debilidades se conviertan en fortalezas. Por tanto, nuestro estudio se basa en conocer las motivaciones y los intereses del grupo de alumnos mayores que en estos momentos están realizando los cursos de informática en el programa universitario de la Universitat Oberta per a Majors (UOM), así como aquellas dificultades con las que los docentes se encuentran de manera más habitual, comparándolo a su vez con un grupo de alumnos que no se han matriculado nunca en un curso de informática.

Palabras clave: persona mayor, nuevas tecnologías, programas universitarios y educación 


\section{Introducción}

El 2012 ha sido el Año Europeo del Envejecimiento Activo y de la Solidaridad Intergeneracional. Como profesionales que estamos en contacto con el colectivo de personas mayores, es nuestro deber reflexionar sobre el estado del envejecimiento en estos momentos. Desde hace ya unos años hasta nuestros días, se habla mucho de envejecimiento activo; es como si ahora mismo fuera la novedad y lo más apropiado fuera incluir este concepto en todas las actividades dirigidas al colectivo de mayores. Nos encontramos en el momento más adecuado para poder pensar y reflexionar sobre lo que quieren decir envejecimiento y activo por sí solos, por separado, para luego darle el significado conjunto que merece. Si entendemos el binomio envejecimiento activo como aquel que posibilita que la persona mayor forme parte importante de la sociedad y que participe de todas sus actividades, podemos afirmar que los programas educativos para mayores están contribuyendo muy positivamente a este fin.

Al respecto, uno de los objetivos de la Universitat Oberta per a Majors (UOM) de la Universitat de les Illes Balears (UIB) es contribuir a este envejecimiento activo desde la participación del alumnado mayor en el desarrollo de actividades que generen esta parte activa. Lo que más nos importa es que el alumnado sea el responsable de su propio proceso de aprendizaje. Ellos por sí mismos han de ser capaces de descubrir el potencial que tienen y, por este motivo se les debe ofrecer actividades útiles y mostrarles esa utilidad vital que pueden tener para su día a día.

Las tecnologías de la comunicación y la información (TIC) pueden ser consideradas como una de las áreas más importantes en lo que se refiere a la participación de los mayores en la sociedad. Las TIC son una forma de posibilitar el acceso a la información y a la interacción social, cultural o educativa, aspectos necesarios para poder desarrollar al máximo nuestras facultades. Las TIC van evolucionando a una velocidad vertiginosa y esta adaptación, a veces, es algo complicada. La brecha que se abre entre las personas con acceso eficiente a las TIC y las que no es la llamada brecha digital.
Un aspecto a tener en cuenta es lo que se conoce como brecha digital de género, según la cual las mujeres encuentran más dificultades de acceso que los hombres a la sociedad de la información. Por otra parte, hay que añadir que la brecha de género se intensifica a través de las diferencias socioeconómicas, de edad y nivel educativo. Cabe en este punto recordar la relación entre género y envejecimiento, que implicaría una doble brecha: la de género y la de edad. Como señalan Arber y Ginn (1996), el género y el envejecimiento están estrechamente conectados en la vida social, de modo que cada uno solo puede entenderse por completo en relación con el otro. A medida que cumplimos años, el contexto social, cultural, económico y político que prevalece en las distintas épocas de nuestra vida influye sobre nosotros. Se plantea, por tanto, la necesidad de potenciar un uso de las TIC que tome como punto de partida el empoderamiento y la capacitación de las mujeres y, más en concreto, de las mujeres mayores con el propósito de avanzar en la igualdad de género y las posibilidades de desarrollo personal, educativo y social (Macías y otros, 2010).

Un aspecto interesante para los profesionales que trabajamos con el colectivo de mayores es conocer qué motivos tienen nuestros alumnos para querer e-incluirse en el mundo de las nuevas tecnologias. En un estudio realizado por Mas y otros (2008) nos hicimos la siguiente pregunta: ¿qué nos dice la literatura acerca de la motivación y los factores que en ella influyen?, la mayoría de los estudios con adultos mayores se centran en conocer los niveles de motivación de este alumnado y la influencia en los mismos de variables de tipo sociodemográfico como la edad, el sexo, la jubilación o el nivel educativo previo. Los estudios coinciden en destacar el alto nivel de motivación de este grupo de estudiantes y en que esta no depende de la edad (Orte, March y Vives, 2007). Los resultados de Sancho y otros (2002) mostraron altos niveles de motivación en este tipo de alumnos, que se manifiestan, entre otras cosas, en el interés que muestran por los cursos, la satisfacción que les produce seguir en ellos, la regularidad con la que asisten a las clases, con escasas ausencias a ellas, y en la valoración de la probabilidad de seguir estudiando. 


\section{Método}

Nuestro objetivo fue realizar un estudio piloto sobre los motivos que mueven al alumnado del programa universitario para mayores de nuestra universidad a matricularse, o no, a los talleres de informática que se ofrecen en nuestro plan de estudios y aquellas principales dificultades o problemas con las que se encuentran una vez iniciado el proceso de aprendizaje.

La muestra estaba formada por 61 alumnos del programa universitario para mayores de la Universitat de les Illes Balears, conocido como UOM (Universitat Oberta per a Majors), divididos en 35 alumnos matriculados actualmente a los talleres de informática y 26 alumnos que no han cursado ningún taller de informática en su proceso de aprendizaje. El rango de edad para los alumnos participantes abarcaba desde los 56 a los 86 años, con una media de edad de 65,76 . El 66,75\% eran mujeres y el 33,25\% hombres. La mayoría de ellos están jubilados o prejubilados $(71,4 \%)$ y tienen un nivel de estudios de bachiller (51,05\%, el 31,85\% elemental y el $19,2 \%$ superior), grado medio (15,2\%) o estudios universitarios $(15,65 \%)$.

La mayoría de los alumnos del taller encuestado tienen ordenador en casa (97\%), aunque un $14,7 \%$ no lo utilizaba antes de los cursos y un $8,8 \%$ se compró el ordenador después de haber empezado a asistir a los talleres de informática. Además, más de la mitad de los alumnos lo utilizan de forma diaria $(58,8 \%)$.

Se elaboraron dos cuestionarios, con preguntas cerradas y preguntas abiertas, por un lado, un primer cuestionario que fue pasado a los alumnos del taller de informática que se estaba realizando en ese momento, por otro lado, un segundo cuestionario que fue pasado a una selección de alumnos que no habían realizado ningún taller de informática. Los participantes lo rellenaron individualmente y de manera voluntaria. Una vez recogidos los cuestionarios, se eliminaron de la muestra aquellos que no estaban cumplimentados en su totalidad. El primer cuestionario se componía de 7 secciones repartidas de la siguiente manera:

1. Variables sociodemográficas (género, edad, estado civil, nivel de estudios y situación laboral).
2. Experiencia que ha tenido el alumnado con los ordenadores (si ha utilizado ordenadores en el trabajo, si dispone de ordenador en casa, de qué tipo de ordenador dispone y con qué frecuencia lo usa).

3. Talleres realizados (qué tipo de talleres ha realizado y qué tipo de talleres le gustaría realizar)

4. Motivaciones e intereses del alumnado.

5. Dispositivo de entrada (uso del ratón, de los botones y del teclado).

6. Uso del ordenador (identificación de las dificultades que tiene el alumnado a la hora de utilizar el ordenador).

7. Sentimiento al utilizar el ordenador.

El segundo cuestionario se componía de 4 secciones repartidas de la siguiente manera

1. Variables sociodemográficas (género, edad, estado civil, nivel de estudios y situación laboral).

2. Experiencia que ha tenido el alumnado con los ordenadores (si ha utilizado ordenadores en el trabajo, si dispone de ordenador en casa, de qué tipo de ordenador dispone y con qué frecuencia lo usa).

3. Posibles talleres realizados (qué tipo de talleres ha realizado y qué tipo de talleres le gustaría realizar).

4. Motivaciones e intereses del alumnado.

\section{Resultados}

\subsection{Motivaciones y uso}

En el cuestionario, se dejó el tema de las motivaciones como una pregunta abierta para no limitar las respuestas de los alumnos. Aun así, las motivaciones que expresaron se pueden englobar y clasificar en las categorías que ya encontraron Mas y otros (2008):

- Para aprender

- Para estar al día y conocer cosas nuevas

- Para estar activo/a

- Para relacionarse

- Para recordar cosas 
- Para estudiar lo que no pudieron cuando eran jóvenes

- Para compartir conocimientos con otras personas

- Para ocupar el tiempo

Las respuestas más frecuentes fueron la motivación de aprender, recordar, ampliar y actualizar conocimientos para poder «utilizar más opciones», «estar más seguro cuando utilizo el ordenador»y «superar las barreras por desconocimiento», "ganar soltura para desenvolverme en la red», «la comodidad para buscar información» o simplemente por «satisfacción personal» o porque «me gusta aprender». Los comentarios respecto a estar al día y conocer cosas nuevas también fueron muy numerosos, (para «vivir en el mundo actual», «el temor a un desfase social y personal»), aunque alguno admitió que «las tecnologías van más deprisa que yo».

Los alumnos también valoraron muy positivamente el uso de las TIC para comunicarse, estar relacionados e intercambiar y compartir conocimientos con otras personas, por lo que el $100 \%$ de los encuestados tiene correo electrónico y lo utiliza con frecuencia el $73.5 \%$ siendo sus contactos principales sus familiares, amigos y compañeros de clase. Un poco más que la mitad del grupo (51\%) utilizaba algún tipo de red social con amigos y familiares y en menor medida (20\%) escribían en algún blog o utilizaban un chat (9\%). Otros comentarios recogidos hacían mención a la instantaneidad de la comunicación, la capacidad de archivo en poco espacio, el entretenimiento que ofrecían las Tic o el tener la mente ocupada.

Los usos que hacían del ordenador eran sobre todo, para obtener información, ocio, comunicarse y participar a través de Internet, comprar por la red $(48,5 \%)$ o poder expresarse creativamente ya fuera en forma de escritura de textos, haciendo presentaciones de temas de su interés o utilizándolo para escribir documentos de trabajo.

La respuesta más frecuente que los alumnos que no han realizado ningún taller o curso de informática expresaron como motivo por el cual no han realizado ningún tipo de taller relacionado con temas tecnológicos fue por falta de tiempo o no tener la oportunidad de hacerlo en este momento, seguido de la respuesta de «no estar motivado por el tema», ya que «me aburre», «no tengo un interés especial» o lo perciben como «un aprendizaje no necesario para su proceso personal». Otras respuestas, en menor porcentaje, que podemos destacar son que han aprendido a usar el ordenador de manera autodidacta o por cuenta propia o que no han realizado ningún taller por no disponer de ordenador.

En cuanto a qué motivaciones o intereses muestran en relación a los temas tecnológicos, expresaron los siguientes: la respuesta más frecuente, en relación a la motivación, fue el hecho de poder obtener y acceder a la información de manera generalizada y posibilitar la actualización de los conocimientos a su vez, las nuevas tecnologías son percibidas como algo necesario para la vida de hoy en día, tanto por el hecho de conocer cosas nuevas, aprender y estar al día como por la posibilidad de estar en contacto con otras personas y lugares, aunque tenemos que destacar que una parte de las respuestas expresaron que no tienen ninguna motivación por el tema. Si nos fijamos en las respuestas sobre los intereses, podemos destacar el hecho de tener un acceso a la información de manera inmediata (leer la prensa por Internet, buscar información específica, etc.) $\mathrm{y}$, de igual manera, el hecho de estar al día de los avances tecnológicos. Comentar que ha ocurrido lo mismo que con las motivaciones: un porcentaje de respuestas expresaron que no tienen ningún interés en este tipo de temas.

\subsection{Dificultades}

El entorno donde se realiza la clase son salas de ordenador con Windows $\mathrm{XP}^{\mathrm{TM}}$ con cañón de proyección desde la máquina del profesor. Dependiendo del taller, los alumnos comparten ordenador. Ahora bien, son curiosos los comentarios que realizan los alumnos a este respecto, desde la inconformidad de aquellos alumnos que solicitan un ordenador para trabajar de forma individual «para practicar lo que dice el profesor» hasta aquellos que comentan «[..] nos va bien ser dos, porque mientras [el compañero] lo hace, yo tomo notas» o «Como [el compañero] sabe más que yo, me deja controlar el ordenador pero me ayuda si tengo dudas». 
Uno de los primeros obstáculos con los que se encuentra el alumno es no reconocer su Escritorio y las diferentes versiones de los programas con los que se trabaja en los talleres respecto a las de su casa $(58,8 \%)$. Después, al trabajar con un usuario que no tienen permisos de administrador, no podemos adaptar el tamaño de la letra, el contraste y otros aspectos que facilitan la lectura y la visualización, pero es importante que conozcan estas características.

A pesar de observar el difícil manejo del ratón por parte de algunas personas en sus primeros contactos con el ordenador (sobre todo cuando tienen temblores en las manos), una vez acostumbrados a los dispositivos, encuentran ligeramente más complicado la utilización del teclado, especialmente la obtención de los símbolos que requieren combinación de teclas o la escritura de acentos.

Las dificultades con las que los alumnos se encuentran con mayor frecuencia son: en cuanto al uso del ordenador, las respuestas expresadas fueron la falta de conocimientos técnicos y de los programas, no saber qué hacer cuando surge algún problema (se bloquea el ordenador, no responde, aparecen mensajes que no entiendes, etc.), en cuanto al uso de Internet, la mayor parte de los alumnos expresaron que es muy difícil su uso, una parte porque «no tengo conocimientos sobre el tema» $\mathrm{O}$ «no conozco el manejo de las tecnologías». Otro problema que podemos destacar es la dificultad en buscar y seleccionar los datos que les interesan, debido a la gran cantidad de información que aparece tras realizar una búsqueda, dificultando el discernir entre información de calidad o no. Si nos centramos en las dificultades en el uso del correo electrónico, las respuestas más frecuentes por parte de los alumnos asistiendo a los talleres tenían que ver sobre todo con adjuntar documentos, los problemas que presentaban el resto eran la dificultad de no entender el funcionamiento y perderse con todas las opciones que se presentan al abrir el correo electrónico, aunque hay un porcentaje muy elevado de alumnos de este segundo grupo que no tiene cuenta de correo electrónico ni interés en crearse una cuenta de correo. Por último entre las dificultades que indicaron en relación al uso del Office ${ }^{\mathrm{TM}} \mathrm{y}$ del sistema operativo del ordenador, un gran número de encuestados en el taller de informática resalta la dificultad de la diversidad de opciones que ofrecen los programas, dificultando la memorización y aumentando la inseguridad que sienten por no dominar el tema. Los alumnos que nunca han participado en talleres respondieron de manera más frecuente que les falta práctica en el Word ${ }^{\mathrm{Tm}} \mathrm{y}$ en otros programas y de una manera muy generalizada indicaron que lo usan o lo utilizan poco, aunque no lo entienden mucho, a pesar de que disponen casi en un $100 \%$ de ordenador en su casa.

\section{Discusión}

En este artículo hemos querido analizar un caso de estudio sobre las motivaciones que impulsan o no a los alumnos de programas universitarios para Mayores (PUM) a apuntarse a talleres de informática y las principales dificultades con las que se encuentran cuando trabajan con el ordenador.

Los resultados demuestran que la mayoría de alumnos tiene ordenador en su casa y que las dificultades generales son compartidas tanto por aquellos alumnos que no han participado en talleres de informática como por los que sí han asistido alguna vez a alguno de los talleres. Ahora bien, a medida que los alumnos aprenden más, los problemas que se presentan son más específicos.

En cuanto a la motivación intrínseca de los alumnos que han entrado contacto con las TIC, podemos apreciar que la formación tecnológica es percibida como necesaria para la vida cotidiana y su utilización es de gran ayuda para posibilitar la comunicación con otras personas, sobre todo con la familia y amigos. De esta manera se sienten con ganas de aprender cosas nuevas. En cambio, el grupo de alumnos que no han tenido contacto con el mundo tecnológico o lo han tenido en un ambiente laboral muy concreto perciben las tecnologías como algo que no les ayudará en su día a día, en su vida personal. Por lo tanto, no les motiva para matricularse a este tipo de cursos.

Ahora nuestro esfuerzo debe recaer en buscar la mejor manera de motivar a este grupo de alumnos e incluirlos en la sociedad tecnológica. 


\section{Referencias}

Arber, S. y Ginn, J. (1996). Relación entre género y envejecimiento: un enfoque sociológico. Madrid: Narcea.

Macías, L., Orte, C. y García-Paredes, A. (2010). La incorporación de las Tic en la programación académica de los Programas Universitarios para Mayores. Trabajo presentado en las II Jornadas sobre Mayores y Nuevas Tecnologías, celebrado en Castellón de la Plana en abril del 2010.

Mas, C., Macías, L., Orte, C. y Fernández, C. (2008). Estudio piloto sobre motivación y aprendizaje en alumnos de primer curso de un programa universitario para mayores. Póster presentado en V Congreso Internacional de Psicología y Educación, celebrado en Oviedo en abril del 2008.

Orte, C., March, M. X. y Vives, M. (2007). Social support, quality of life and University Programmes for Seniors. Educational Gerontology, 33(11), 995-1013.

Sancho, C., Blasco, M. J., Martínez-Mir, R. y Palmero, F. (2002). Análisis de la motivación para el estudio en adultos mayores. Revista electrónica de motivación y emoción, 5(10). Disponible en: http://reme.uji.es. 